\title{
Nanophotonics Sensor Based on Microcantilever for Chemical Analysis
}

\author{
Wenfeng Xiang and Chengkuo Lee, Member, IEEE
}

\begin{abstract}
A Si-based cantilever sensor with photonic crystal (PC) resonator as readout for chemical sensing and analysis has been developed. The resonant wavelength shift of $\mathrm{PC}$ resonator is resulted from PC deformation induced by cantilever bending, in which this optical readout scheme facilitates cantilever deflection measurements in liquid. Through numerical simulation, we demonstrate that the detection capability of this micromechanical sensor operated in water is better than that of sensor operated in air. The minimum detectable $Z$-displacement and strain of $\mathrm{Si} / \mathrm{SiO}_{2}$ cantilever sensor are derived as $0.6 \mu \mathrm{m}$ and $0.0098 \%$ in water and $0.812 \mu \mathrm{m}$ and $0.0144 \%$ in air, respectively. This novel micromechanical sensor shows its promising future in applications such as detection of proteins and DNA in solution.
\end{abstract}

Index Terms-MEMS, microcantilever, nanomechanical sensor, NEMS, photonic crystal resonator.

\section{INTRODUCTION}

I $\mathrm{N}$ THE development of novel chemical and biological sensors, micro/nanoelectromechanical systems (MEMS/ NEMS) have received a lot of attention because of the superior detection capability for chemical analytes like cells, virus, DNA molecules, and proteins. Cantilever beam with dimension in micro/nanometer scale is a well-adopted sensor structure. The main feature distinguishing it from other sensor structures is its high sensitivity to cantilever deflection, i.e., strain. When molecular adsorption is confined to one side of a microcantilever, it undergoes deflection due to a differential surface stress caused by the forces involved in the adsorption process [1]. By monitoring the variation of cantilever deflection resulted from the adsorptioninduced stress, the chemical analytes can be measured with high sensitivity. The detection schemes of cantilever deflection could be mainly categorized as electrical [2]-[8] and optical [9]-[11] approaches. Compared to the electrical detection schemes, the so-called optical level method has been commercialized with many advantages, such as linear response, simplicity, and excellent reliability.

Photonic crystals (PCs) have found important applications ranging from lasers, waveguides, filters, and modulators to sensors [12]-[15]. In two-dimensional (2-D) PCs, the electromagnetic field is localized in the low refractive index region, i.e., air holes. The resonant cavity structures can be created by

Manuscript received January 4, 2009; revised February 12, 2009. First published May 19, 2009; current version published October 7, 2009. This work was supported by the National University of Singapore under Grant No. R-263000-475-112 and A-STAR, SERC Grant No. 0921010049.

The authors are with the Department of Electrical and Computer Engineering, National University of Singapore, 4 Engineering Drive 3, Singapore 117576 (e-mail: elexw@nus.edu.sg; elelc@nus.edu.sg).

Color versions of one or more of the figures in this paper are available online at http://ieeexplore.ieee.org.

Digital Object Identifier 10.1109/JSTQE.2009.2016578 introducing point and/or line defects into 2-D PC periodic holes array in which it supports field localization in photonic bandgap. Thus, PC resonators exhibit resonant wavelength peak, which is a function of the surface state of the defects or the shape and dimension of defects in the PC resonant cavity structures. In our previous study, the detected mechanism of microcantilever sensor with PC nanocavity resonator has been reported [16], [17]. In this paper, we investigate the influence of the effective refractive index on the performance of the microcantilever sensors by analyzing the different kinds of sensors and different detected environment.

\section{DEVICE DESIGN}

As shown in Fig. 1(a), in this paper, we describe a microcantilever sensor with an integrated PC resonator at the edge of silicon-on-insulator (SOI) chip as a strain sensor, i.e., an optical readout. In PCs, defect cavities give rise to a defect state in the photonic bandgap. By varying the spacing of these defect cavities, we can tune the resonant wavelength of this state across the bandgap of the PC resonator. In our design, this PC resonator consists of a U-shaped $\mathrm{Si}$ waveguide encompassed by air holes and four-hole along the input waveguide, i.e., the resonant cavity (shown in inset). Defect length $A_{d}$ is defined as the distance between the second and third hole of said four-hole in the resonant cavity and is given by

$$
A_{d}=2 a-2 r
$$

where $a$ is the lattice constant of PC and $r$ is the radius of air holes in PC. Fig. 1(b) shows that the two microcantilevers of different dimensions can be fabricated in a fluidic channel. Solution containing molecules flow through this channel such that the targeted molecules are bound on to cantilever surface due to the biomolecule selective binding, e.g., antigens to antibodies. The induced cantilever deflection is characterized in terms of the resonant wavelength shift due to deformation of PC resonator. Moreover, optical fibers can be easily coupled to the input and output waveguide terminals outside the fluidic channel. Each one of the plural microcantilevers is designed for detection of a specific molecule, while multiple unknown molecules could be tested in the same fluidic channels according to coated counterpart probes on one of these cantilevers. The primary advantage of proposed device is that it can be operated in a broad range of environments such as gas and solution. Additionally, these structures can be fabricated by using deep UV lithography, dry and wet etching, and photosensitive polymers as the fluidic channel walls. Thus, it allows easy integration of microelectronics, microfluidics, or other photonic elements in one device. 

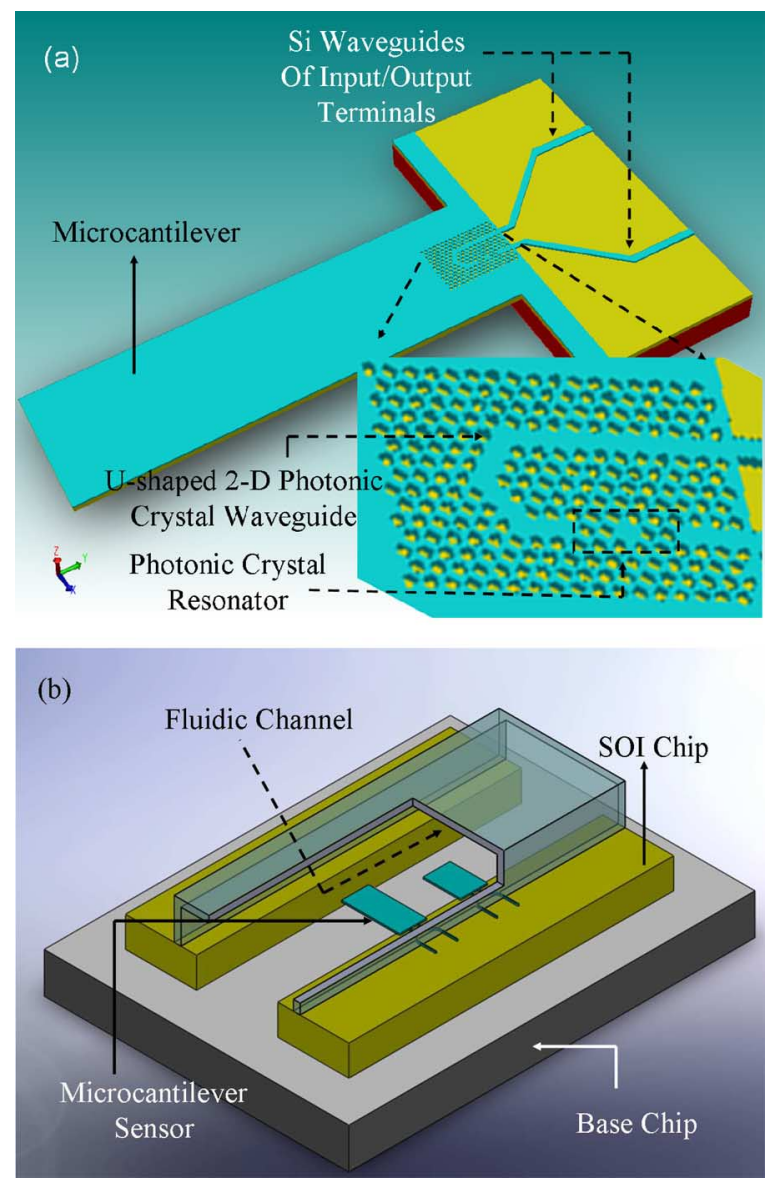

Fig. 1. (a) 3-D illustration of a microcantilever sensor using PC resonator as a strain sensor located at the edge of SOI chip. Inset shows the 3-D illustration of the PC resonator. (b) Conceptual drawing of two microcantilevers packaged inside a fluidic channel. These two microcantilevers consist of PC resonator as the readout, such that these novel microcantilever sensors could be densely arranged in fluidic channel for detection of various types of biomolecules due to selective binding.

\section{RESULTS}

In this study, we investigated two kinds of microcantilevers. They are the $\mathrm{Si} / \mathrm{SiO}_{2}$ bilayer microcantilever and Si microcantilever. The length and width of cantilevers are $50 \mu \mathrm{m}$ and $15 \mu \mathrm{m}$, respectively. The layer thicknesses of $\mathrm{Si}$ and $\mathrm{SiO}_{2}$ layers are 0.25 $\mu \mathrm{m}$ and $0.6 \mu \mathrm{m}$, respectively. We deploy the device layer and insulating oxide layer of SOI substrate as the aforementioned $\mathrm{Si}$ and $\mathrm{SiO}_{2}$ layers. The $\mathrm{PC}$ resonator comprises periodic holes array etched down to $\mathrm{SiO}_{2}$ layer. The hexagonally arranged air holes with radius and pitch of $0.22 \mu \mathrm{m}$ and $0.54 \mu \mathrm{m}$ and the $A_{d}$ of $0.64 \mu \mathrm{m}$ are selected based on the bandgap diagram simulation result by using 2-D finite-difference time-domain (FDTD) method. The finite-element method (FEM) is deployed to get the deformation contour of holes of PC resonator under force loads. The Young's modulus and Poisson's ratio of $\mathrm{Si}$ and $\mathrm{SiO}_{2}$ are $130 \mathrm{GPa}, 70 \mathrm{GPa}, 0.3$ and 0.17 , respectively. The refractive indices of $\mathrm{Si}, \mathrm{SiO}_{2}$, air, and water are $3.46,1.46,1$, and 1.33 , respectively. The deformation data of $\mathrm{PC}$ resonator under different loading force are provided in the FDTD simulation.

Fig. 2(a) shows the simulated various resonant peaks of the $\mathrm{Si} / \mathrm{SiO}_{2}$ cantilever sensor operated in water under different force
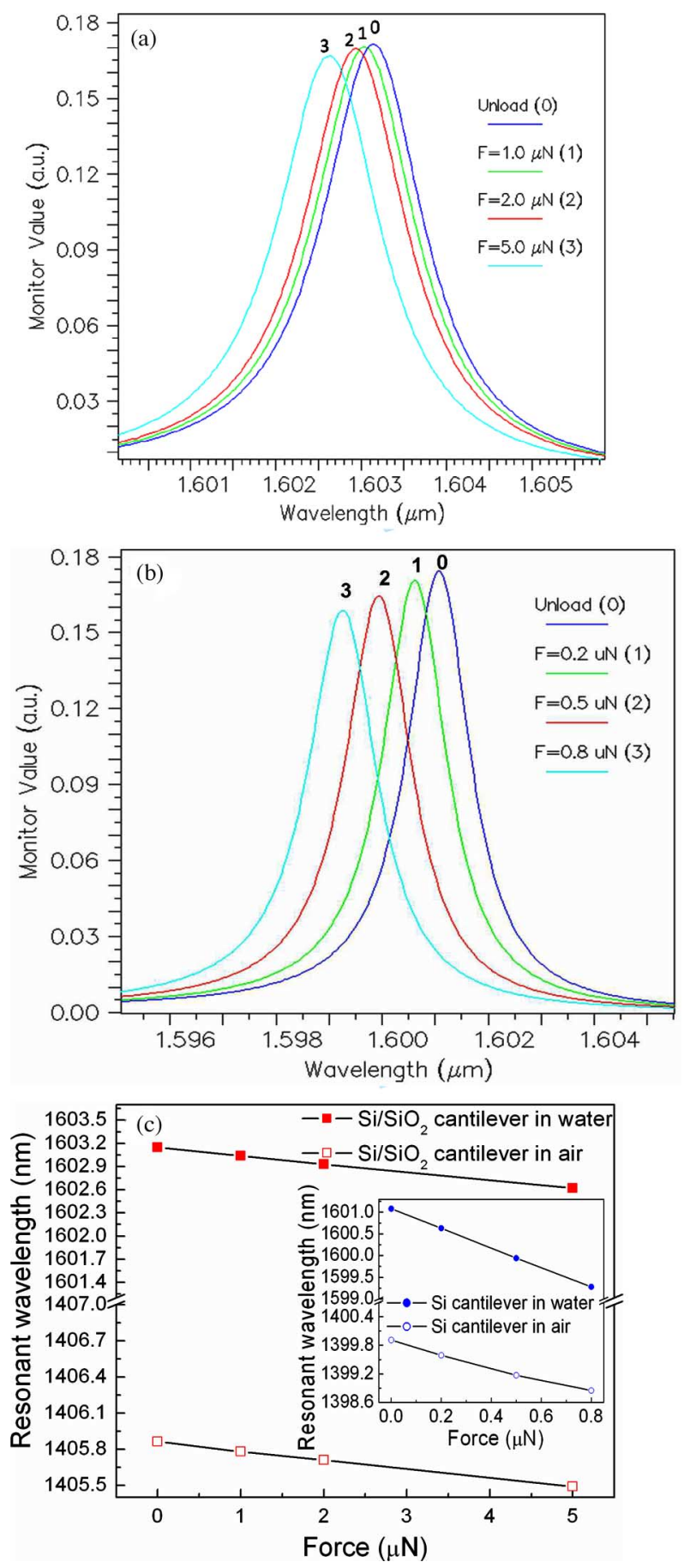

Fig. 2. (a), (b) Output resonant peaks of the $\mathrm{Si} / \mathrm{SiO}_{2}$ cantilever sensor and $\mathrm{Si}$ cantilever sensor operated in water under different loading force; (c) the resonant wavelength of the $\mathrm{Si} / \mathrm{SiO}_{2}$ cantilever and $\mathrm{Si}$ cantilever operated in air/water as a function of loading force (inset).

loads at the cantilever end from 0 to $5 \mu \mathrm{N}$. Fig. 2(b) shows the same trend of resonant peak shift of the Si cantilever sensor operated in water with the loading force from 0 to $0.8 \mu \mathrm{N}$. Fig. 2(c) shows the wavelength of resonant peaks of these two kinds of microcantilevers operated in air/water as a function of the loading force. Linear relationship is observed for the variation of resonant peaks regarding the loading force. The resonant peaks of both kinds of cantilever sensors have a blue shift with the 


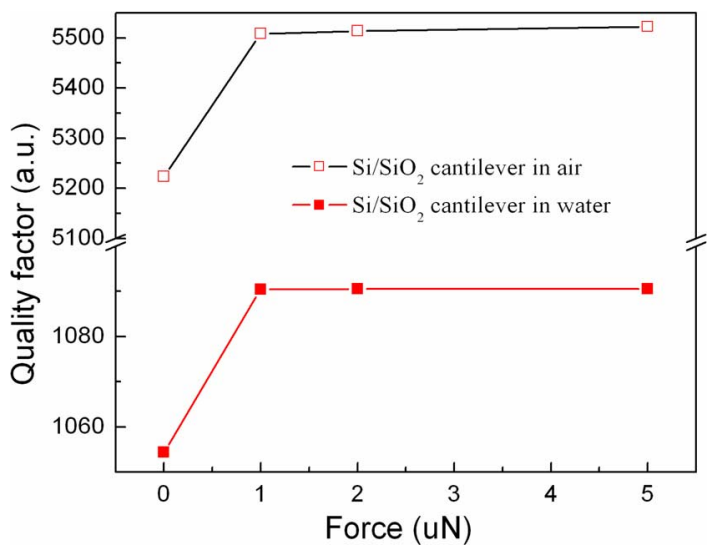

Fig. 3. Quality factor change of the resonant peaks of the $\mathrm{Si}_{\mathrm{SiO}}$ cantilever sensor operated in air/water under different loading force.

loading force increasing, corresponding to the increase of $A_{d}$ in the PC resonator. The resonant peaks of the $\mathrm{Si} / \mathrm{SiO}_{2}$ cantilever sensor and Si cantilever sensor without loading force in air and in water are derived as $1405.8 \mathrm{~nm}, 1603.1 \mathrm{~nm}$, and $1399.9 \mathrm{~nm}$, $1601.1 \mathrm{~nm}$, respectively. In comparison to the initial peaks of the sensors operated in air and in water, peaks shift to longer wavelength region, i.e., the red shift is observed. Moreover, the initial wavelength of resonant peaks of Si cantilever sensor are shorter than that of the $\mathrm{Si} / \mathrm{SiO}_{2}$ cantilever sensor. These results indicate that the resonant wavelength increases with the effective refractive index of the PC resonator increasing. Fig. 3 shows the variation of quality factor of resonant peaks of the $\mathrm{Si} / \mathrm{SiO}_{2}$ cantilever sensor operated in air/water as the function of the loading force. As shown in Fig. 3, the loading force has no significant influence on the quality factor. However, the quality factor of the $\mathrm{Si} / \mathrm{SiO}_{2}$ cantilever sensor operated in air is larger than that of the $\mathrm{Si} / \mathrm{SiO}_{2}$ cantilever sensor operated in water. It indicated that the increment of effective refractive index decreases the quality factor of resonant peaks.

By combining the FEM results under various force loads with the FDTD data, the resonant wavelength shift is plotted as the function of the $Z$-displacement at the end of cantilever as shown in Fig. 4 , where the $Z$-displacement means the vertical displacement of cantilever end. The linear relation between the resonant wavelength shift and the $Z$-displacement is observed. Regarding the $0.1 \mathrm{~nm}$ wavelength shift resolution, the minimum detectable $Z$-displacement is about $0.97 \mu \mathrm{m}$ and $0.795 \mu \mathrm{m}$ for the Si microcantilever operated in air/water and $0.812 \mu \mathrm{m}$ and $0.6 \mu \mathrm{m}$ for the $\mathrm{Si} / \mathrm{SiO}_{2}$ microcantilever operated in air/water, respectively. It shows that the better detection limit of $Z$-displacement can be achieved in water than in air for both kinds of microcantilever sensors. Moreover, the minimum detectable $Z$-displacement of the $\mathrm{Si} / \mathrm{SiO}_{2}$ cantilever sensor is smaller than that of $\mathrm{Si}$ cantilever sensor operated in the same environment. These results indicated that the minimum detectable $Z$-displacement of sensors is improved when we compare $\mathrm{Si} / \mathrm{SiO}_{2}$ bilayer cantilever with Si single cantilever.

We have reported that the resonant wavelength shift is mainly attributed to the change of $A_{d}$ [15]. Thus, the $A_{d}$ is the most critical parameter in the proposed $\mathrm{PC}$ resonator. The strain of PC

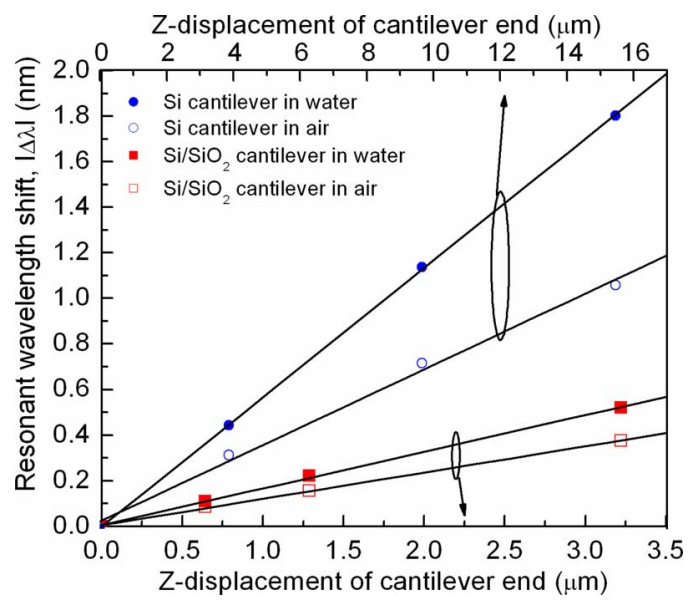

Fig. 4. Resonant wavelength shift versus the $Z$-displacement at the cantilever end of various cantilevers operated in water/air.

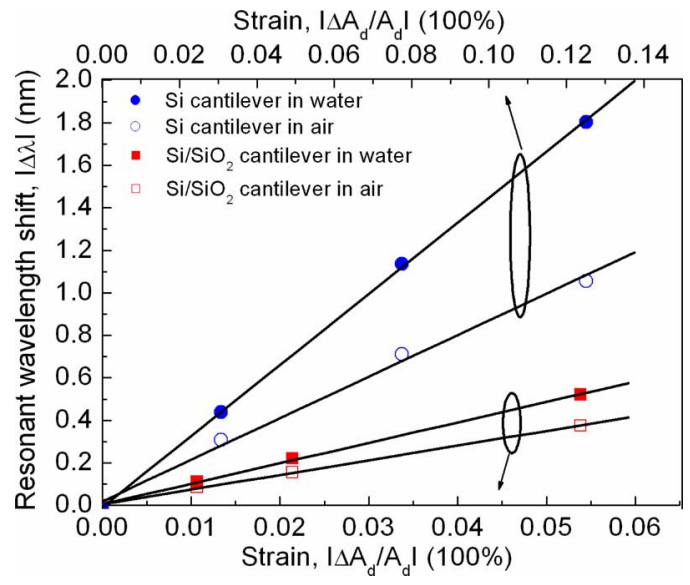

Fig. 5. Resonant wavelength shift versus the strain of PC resonator of various cantilevers operated in water/air.

resonator was defined as a ratio of the change in defect length $\left(\Delta A_{d}\right.$ ) to the initial $A_{d}$. As shown in Fig. 5, the resonant wavelength shift follows the $\Delta A_{d}$ linearly. Again, in terms of $0.1-\mathrm{nm}$ wavelength shift resolution, the minimum detectable strain is about $0.0144 \%$ (in air) and $0.0098 \%$ (in water) for the $\mathrm{Si}_{2} / \mathrm{SiO}_{2}$ cantilever, respectively, while the minimum detectable strain is slightly decreased to $0.0094 \%$ and $0.0069 \%$ in the cases of $\mathrm{Si}$ cantilever operated in air/water, respectively. It can be seen that the strain sensitivity of $\mathrm{Si}$ cantilever sensor is better than that of the $\mathrm{Si} / \mathrm{SiO}_{2}$ cantilever sensor under the same environment. Generally speaking, the softer cantilever gives better strain sensitivity since the strain is inversely proportional to Young's modulus and the square of layer thickness. The $\mathrm{Si} / \mathrm{SiO}_{2}$ bilayer cantilever shows higher stiffness, while it exhibits higher effective refractive index as well. Under the same strain, $\mathrm{PC}$ resonator of higher effective refractive index leads to larger wavelength shift. We observed that the enlarged wavelength shift cannot overcome the reduction of strain change when we compare $\mathrm{Si} / \mathrm{SiO}_{2}$ bilayer cantilever to Si cantilever in Fig. 5. 


\section{CONCLUSION}

We designed the novel Si nanophotonics sensors based on microcantilever. The deformation of PC resonator induced by microcantilever bending can be detected by measuring the resonant wavelength shift in PC resonator. Integrating these microcantilever sensors with PC resonators in a fluidic channel, the targeted molecules can be detected synchronously. The $\mathrm{Si} / \mathrm{SiO}_{2}$ cantilever sensor and Si cantilever sensor operated in air/water are characterized with respect to the various force loads. With the increase in loading force, the resonant peaks of both kinds of cantilever sensors have a blue shift. For the PC resonator, the effective refractive index has a crucial influence on the variation of resonant wavelength. Therefore, the initial wavelength of resonant peaks of both kinds of sensors operated in water are longer than that operated in air, and the initial wavelength of resonant peaks of Si cantilever sensor operated in air/water are shorter than that of $\mathrm{Si} / \mathrm{SiO}_{2}$ cantilever sensor operated in air/water. In addition, the force and strain sensing capability are increased when the microcantilever sensors operated in water. The minimum detectable $Z$-displacement and strain for $\mathrm{Si} / \mathrm{SiO}_{2}$ cantilever are 0.6 $\mu \mathrm{m}$ and $0.0098 \%$ operated in water and $0.812 \mu \mathrm{m}$ and $0.0144 \%$ operated in air. In addition, $0.795 \mu \mathrm{m}$ and $0.0069 \%$ and $0.97 \mu \mathrm{m}$ and $0.0094 \%$ are derived for the Si cantilever sensor operated in water and in air, respectively. For these two kinds of microcantilever sensors, the stiffer one, i.e., the $\mathrm{Si} / \mathrm{SiO}_{2}$ bilayer cantilever, shows the better performance in detection of the smallest vertical displacement (or force), while the softer one, i.e., the $\mathrm{Si}$ cantilever, shows the higher sensitivity in strain detection.

\section{REFERENCES}

[1] N. V. Lavrik, M. J. Sepaniak, and P. G. Datskos, "Cantilever transducers as a platform for chemical and biological sensors," Rev. Sci. Instrum., vol. 75, pp. 2229-2253, Jul. 2004.

[2] M. Tortonese, R. C. Barrett, and C. F. Quate, "Atomic resolution with an atomic force microscope using piezoresistive detection," Appl. Phys. Lett., vol. 62, pp. 834-836, Feb. 1993.

[3] T. Itoh, C. Lee, and T. Suga, "Deflection detection and feedback actuation using a self-excited piezoelectric $\mathrm{Pb}(\mathrm{Zr}, \mathrm{Ti}) \mathrm{O}_{3}$ microcantilever for dynamic scanning force microscopy," Appl. Phys. Lett., vol. 69, pp. 20362038, Sep. 1996.

[4] C. Lee, T. Itoh, and T. Suga, "Micromachined piezoelectric force sensors based on PZT thin films," IEEE Trans. Ultrason., Ferroelectr., Freq. Control, vol. 43, no. 4, pp. 553-559, Jul. 1996.

[5] C. Lee, T. Itoh, R. Maeda, and T. Suga, "Characterization of micromachined piezoelectric PZT force sensors for dynamic SFM," Rev. Sci. Instrum., vol. 68, pp. 2091-2100, 1997.

[6] C. Lee, T. Itoh, R. Maeda, T. Ohashi, and T. Suga, "Development of a piezoelectric self-excitation and self-detection mechanism of PZT microcantilevers for dynamic SFM in liquid," J. Vac. Sci. Technol. B, vol. 15 , pp. 1559-1563, Jul. 1997.

[7] C. Lee, T. Itoh, and T. Suga, "Self-excited piezoelectric PZT microcantilevers for dynamic SFM-with inherent sensing and actuating capabilities," Sens. Actuators A, Phys., vol. 72, pp. 179-188, Jan. 1999.

[8] G. Shekhawat, S.-H. Tark, and V. P. Dravid, "MOSFET-embedded microcantilevers for measuring deflection in biomolecular sensors," Science, vol. 311, pp. 1592-1595, 2006.

[9] G. Meyer and N. M. Amer, "Novel optical approach to atomic force microscopy," Appl. Phys. Lett, vol. 53, pp. 1045-1047, 1988.

[10] K. Zinoviev, C. Dominguez, J. A. Plaza, V. J. C. Busto, and L. M. Lechuga, "A novel optical waveguide microcantilever sensor for the detection of nanomechanical forces," J. Lightw. Technol., vol. 24, pp. 2132-2138, May 2006

[11] A. Llobera, V. J. Cadarso, K. Zinoviev, C. Dominguez, S. Büttgenbach, J. Vila, and J. A. Plaza, "Poly(dimethylsiloxane) waveguide cantilevers for optomechanical sensing," Photon. Technol. Lett., vol. 21, pp. 79-81, Jan. 2009.
[12] H. Benisty, J.-M. Lourtioz, A. Chelnokov, S. Combrié, and X. Checoury, "Recent advances toward optical devices in semiconductor-based photonic crystals," Proc. IEEE, vol. 94, no. 5, pp. 997-1023, May 2006.

[13] C. Monat, P. Domachuk, and B. J. Eggleton, "Integrated optofluidics: A new river of light," Nat. Photon., no. 1, pp. 106-114, Feb. 2007.

[14] D. Erickson, S. Mandal, A. H. J. Yang, and B. Cordovez, "Nanobiosensors: Optofluidic, electrical and mechanical approaches to biomolecular detection at the nanoscale," Microfluid. Nanofluid., vol. 4, pp. 33-52, 2008.

[15] C. Lee, R. Radhakrishnan, C. C. Chen, J. Lin, J. Thillaigovindan, and N. Balasubramanian, "Design and modeling of a nanomechanical sensor using silicon photonic crystals," J. Lightw. Technol., vol. 26, pp. 839-846, Apr. 2008.

[16] C. Lee, J. Thillaigovindan, C.-C. Chen, X. T. Chen, Y.-T. Chao, S. Tao, W. Xiang, A. Yu, H. Feng, and G. Q. Lo, "Si nanophotonics based cantilever sensor," Appl. Phys. Lett., vol. 93, 113113, 2008.

[17] C. Lee and J. Thillaigovindan, "Optical nanomechanical sensor using Si photonic crystals cantilever embedded with nanocavity resonator," Appl. Opt., vol. 48, pp. 1797-1803, Apr. 2009.

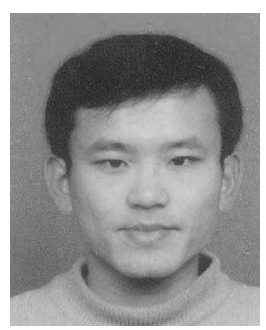

Wenfeng Xiang received the B.Sc. degree from the Department of Physics, Shandong Normal University, Jinan, China, in 2000, and the Ph.D. degree in materials science and engineering from the Institute of Physics, Chinese Academy of Sciences, Beijing, China, in 2005.

Since 2005 to 2006, he worked as a Postdoctoral Researcher at the Department of Materials Science and Engineering, Gwang-Ju Institute of Science and Technology. From 2006 to 2007, he worked as a Research Fellow at the School of Electrical and Electronic Engineering, Nanyang Technological University. He is currently a Research Fellow in the Department of Electrical and Computer Engineering, National University of Singapore, Singapore. His research interests include optical microelectromechanical systems, nanophotonics sensor, and optical communications.

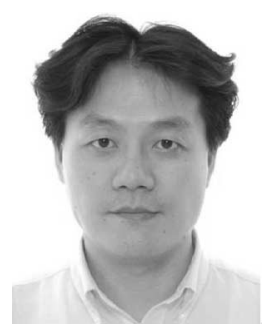

Chengkuo Lee (S'93-M'96) received the M.S. degree in materials science and engineering from National Tsing Hua University, Hsinchu, Taiwan, in 1991, the M.S. degree in industrial and system engineering from Rutgers University, New Brunswick, NJ, in 1993, and the Ph.D. degree in precision engineering from the University of Tokyo, Tokyo, Japan, in 1996.

From 1993 to 1996 , he worked as a Foreign Researcher in the Nanometerscale Manufacturing Science Laboratory, Research Center for Advanced Science and Technology (RCAST), University of Tokyo. He was also a JST Research Fellow in the Mechanical Engineering Laboratory, AIST, MITI, in 1996. Thereafter, he was a Senior Research Staff Member of Microsystems Laboratory, Industrial Technology Research Institute (ITRI). In September 1997, he joined the Metrodyne Microsystem Corporation, Hsinchu, Taiwan, and established the microelectromechanical systems (MEMS) device division and the first micromachining fab for commercial purposes in Taiwan. He was the Manager of the MEMS device division between 1997 and 2000. He was an Adjunct Assistant Professor in the Electrophysics Department of National Chiao Tung University, China, in 1998, and an Adjunct Assistant Professor in the Institute of Precision Engineering of the National Chung Hsing University, China, from 2001 to 2005. He cofounded Asia Pacific Microsystems, Inc. (APM), Hsinchu, Taiwan, in August 2001, and he became the Vice President of R\&D, then later until the end of 2005, the VP of optical communication business unit and Special Assistant of CEO in charge of international business and technical marketing for MEMS foundry service at APM, Inc., one of the top 30 MEMS manufacturers in the world in recent years. Currently, he is an Assistant Professor at the Department of Electrical and Computer Engineering of the National University of Singapore and a Senior Member of Technical Staff at the Institute of Microelectronics (IME), Agency for Science, Technology and Research (A*STAR), Singapore. He has authored or coauthored more than 110 international conference papers and extended abstracts, 60 peer-reviewed international journal articles, and eight U.S. patents in MEMS and nanotechnology field.

Dr. Lee is the member of the Materials Research Society (MRS) and the Institution of Electrical Engineers (IEE) of Japan. 